\title{
Eradication of Malaria
}

\author{
GEORGE MACDONALD, C.M.G., M.D.
}

$\mathrm{I}^{\mathrm{s}}$ S IT POSSIBLE to eradicate malaria, to get rid of it, root and branch, so that it is no longer with us and can be forgotten? For that matter, is it possible to eradicate in the same final manner a host of infections and biological complexes which are harmful to man in one way or another? Virtually the whole history of preventive medicine is based on the concept that one should protect oneself against infections which are inevitably present in the environment and will continue to be present, even though the individual, or groups of individuals, may in some way isolate themselves from the environment and remain untainted. The concept of eradication says that it is unnecessary for the infection to remain in the environment, whether immediate or remote. Through suitable aggressive action, backed by adequate administration, the infection itself can be exterminated so that protective measures are no longer necessary. We owe this new concept to the Rockefeller Foundation and, within the Rockefeller Foundation, largely to Dr. Fred L. Soper, whose initiative and determined action proved that the eradication concept is not only theoretically, but practically possible.

\section{Feasibility of Eradication}

The original demonstration of feasibility was in eradication in Central and South America of Aedes aegypti, the mosquito carrier of urban yellow fever. Against this carrier the logic of

Dr. Macdonald is director of the Ross Institute of Tropical Hygiene and professor of tropical hygiene, London School of Hygiene and Tropical Medicine. literal root-and-branch extermination was applied from 1930 onwards, and under the guidance and control of Soper proved brilliantly successful. The root-and-branch concept postulated that the wide and continued prevalence of this mosquito, which flourished in every town and every compound of every town of tropical America, constituted the branches of a system with roots in certain "mother foci" in specially favorable sites. Within these sites an unbroken continuity of generations provided a constant source from which potential breeding places elsewhere could always be reinvaded after periods of clearance due to natural or humanly operated causes. Soper's contribution was to focus attention on the root, the hidden mother focus. This could be found only after clearance of the proliferating branches and then a most determined search but, once found, could be eliminated with resultant total interruption of the source of fresh infection (1). Success was rapid. Within a year urban yellow fever had come virtually to an end. In subsequent years, A. aegypti has been eradicated from towns and villages of a large part of South and Central America.

Soper and Wilson later applied their rootand-branch concepts in the eradication from Brazil of Anopheles gambiae, an invading mosquito which threatened to make South America

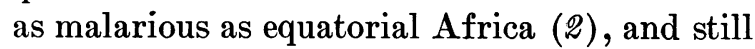
later, in eradication of the same mosquito from parts of Egypt, a country it had invaded with disastrous results (3). Soper has since taken a prominent part in both the practice and promotion of the eradication of malaria by attack on the adult mosquito and has turned both his 
and our attention to the possibilities of eradicating tuberculosis (4), smallpox, and yaws.

The eradication approach, developed by Soper in so many fields, constitutes a complete revolution in our methods of thinking about the management of infectious diseases. Since adoption of it is so largely due to him, I consider the invitation to give a Fred L. Soper Lecture a peculiarly high honor.

\section{Concept of Eradication}

Before reviewing the special problem of the eradication of malaria, it seems worthwhile to consider in more detail the general concept of eradication of any infection and of the factors in transmission which make eradication possible or which may be an impediment to it. My audience is by no means exclusively medical, and perhaps this is to some extent an advantage, since pursuit of the objective entails rejection of many traditional lines of medical approach to the management of disease. An approach is substituted which, while taking fullest possible account of all relevant biological facts, is more typical of the engineer, whose training directs him to study the dynamics of a system and to analyze the factors which permit the indefinite reproduction of a happening. It has been commonly said that to prevent malaria one should think like a mosquito. However, to eradicate it, this is not enough; one should also think like Fred Soper.

The traditional medical and parasitological form to describe the transmission of an infection is in terms of a "cycle" which takes account of all the biological facts and morphological forms in the growth and development of the organism from maturity in one host to the attainment of maturity in another, although characteristically the cycle is represented as if the original host were reinvaded. In many infections this cycle is relatively simple. In others, however, and particularly in infections characteristic of tropical regions, the cycle is increasingly complicated both by the nature of the more highly developed organisms involved and by the occurrence of some external development (whether free-living, in an insect, in a snail, or in a series of hosts) in the process of transmission. In malaria, this cycle includes three en- tirely different mechanisms of multiplication of the parasite in two separate hosts. The elaborations of the mechanisms of these developmental forms constantly being demonstrated have perhaps hypnotized us and distracted our attention from the fact that this cycle explains only a single item of transmission. The cycle conveys few clues as to why the infection persists indefinitely in a community, as the infection must if it is to remain an acknowledged happening.

The cycle is a single link in the transmission of disease. Maintenance of disease transmission demands a successional series or chain of links in which infection is passed from one individual to another. This chain must have remained unbroken, not merely temporarily but continuously for an infinitely long time, to have allowed the infection to survive. Control calls for breaking a single link of the chain to protect an individual. Eradication calls for examining the mechanism of the infinitely long chain to see what factors make indefinitely continued reproduction possible and how the endless chain can be broken. The individual cycles, of course, are important, but not because of the morphological characteristics of the parasite or its insect host but because of the quantitative characteristics which have a bearing on the probability of maintenance of an indefinite succession of transmission.

In all infections this has two facets. On the one hand, there is the host-parasite relationship which determines essentially the duration of infectivity and the development of immunity and which can be taken as fairly standard for any group of cases. On the other hand, there is the environment, which determines the scale of reproduction, a scale, therefore, having as many values as there are types of environment.

In both simple and complicated infections, indefinite maintenance of reproduction is an exacting demand. The environment should remain permanently favorable, and interruptions in transmission should never exceed a certain time limit, dependent on the survival of the organism. Moreover, since infections always immunize their recipients to some degree, maintenance requires that transmission should be continued in communities where the number of nonimmunes is constantly and adequately replenished, typically by births. There is thus a 
minimum size of population which can maintain an infection, and in some instances this minimum size is fairly large indeed.

Any change, natural or artificial, which interrupts transmission or breaks a community into units too small to support the infection inevitably ends the succession of cases in the area concerned. Since these happenings are far from uncommon, the natural history of infection or of any biological complex usually includes the restriction of this continued chain of transmission to certain localities where conditions are particularly favorable. These localities may be small and widely separated, but it is from them that the infection diffuses to invade the community as a whole when conditions become generally favorable.

Such happenings occur in almost any biological complex. It follows that if the general distribution of an infection can first be brought under effective restraint and the primary foci then sought out and controlled, the whole complex can be terminated. This was the way that A. aegypti was eradicated from South American towns and A. gambiae from Egypt and Brazil.

Exactly the same considerations apply to any infection, but the world has been slow to recognize the possibility of their applications. We may rationally consider the eradication of any infection in which we are capable of preventing a single case. We shall need programs based on biological knowledge but consisting essentially of formulation of a definite objective, creation of an administrative system, and operation over an adequate geographic area of a specific campaign, designed first to prevent general proliferation and subsequently to seek out and destroy the mother foci.

For the most part, in such campaigns we would use carefully standardized techniques. The campaigns, however, should be under the closest technical check. They should incorporate a mechanism whereby problems encountered in the field could be rapidly transferred to a research group, whose findings could be rapidly incorporated into field practice. These operational principles can be applied to malaria, tuberculosis, typhoid, venereal disease, whooping cough, or to any other infection, whether temperate, cosmopolitan, or tropical. The time will come when we will talk of eradication in all these contexts, and we will rid ourselves, literally, one by one, of all these specific infections.

\section{Attack on Malaria}

In the meantime these principles of fighting disease are being applied to the eradication of malaria. Certainly it is not the easiest infection to eradicate, though it is incomparably the most important. In the innumerable estimates of the significance of malaria, all have agreed on the immensity of its impact. The comments, however, have been divergent because of the enormous variation in the characteristics of the disease in different epidemiologic zones of the world and because of the impossibility of assessing in material terms the effects of a disease which has caused several million deaths a year, made ill health the primary characteristic of infancy and early childhood over vast areas, and caused immeasurable interference in the productivity of the adult population. Sudden application of fully effective prevention in Ceylon resulted in reduction of the general death rate by 40 percent within 1 year (5) and later permitted recolonization of about half the island which had previously been almost uninhabited. That the zones of the world most severely affected by malaria, equatorial Africa and the IndoChinese area, are perhaps the most retarded in development is no coincidence. Almost the only parts of the world in which no highly developed culture or civilization has ever arisen and which remain undeveloped to the present day are the areas severely affected by malaria.

Eradication of malaria by using residual insecticides had been attempted and had even been achieved in several countries or large regions before the general acceptance of the eradication concept in 1955. The United States of America, Venezuela, British Guiana, Greece, Crete, and some other Mediterranean areas already had highly developed programs before 1955 . Before that year, however, the general concept was one of continued control, which, like all previous measures, was expected to continue indefinitely.

A setback in malaria control that threatened to imperil all previous progress awakened people to the possibilities in eradication. The mos- 
quito became resistant to the insecticides then in use, first in Greece, then in Nigeria and half a dozen other places, till it seemed as if this resistance might spread and totally frustrate progress in control and even cause a reversion to the pre-insecticide era. The Eighth World Health Assembly, reviewing this situation, decided on an all-out effort before it was too late, and thus organized eradication came into being. It might have been well to have started, simultaneously, an intensive research study of resistance, its nature, causation and, if possible, its prevention. Such a study was not, however, at that time within the capacities and functions of the World Heath Organization, and research had to await private initiation. Nearly 18 months later the challenge for research was first met. The Ross Institute of Tropical Hygiene of London made a considerable contribution to this research effort $(6)$. Nevertheless the delay and the inevitably small scale on which research was first undertaken fully justified the subsequent entry of the Organization into malaria research and also justified the Organization's proposals to bring some elements of research under WHO control. Then, in predicaments such as the one of resistant pesticides, WHO could inject the urgency into research which operational problems might demand.

\section{WHO Eradication Schemes}

No formal technical plans for achievement of eradication through deliberate schemes then existed. Such schemes were subsequently prepared by the WHO Expert Committee on Malaria and are described in a series of WHO reports $(y-11)$. They were inevitably exploratory but from the first followed the general concepts applied earlier by Soper and his colleagues in allied fields. The skill and determination with which the Organization has applied these schemes deserve high praise. The difficulties were, however, inevitably great. The Organization itself had no power to initiate programs. This responsibility rested with the individual countries. Nor could the Organization, being limited by its terms of reference to technical aid, finance the programs. Material resources had to come from the countries themselves, assisted mainly by international and bilateral aid sources. The stimulation and integration of activity in eradication therefore presented diffculties. Delay, however, cannot often be laid at the door of the Organization.

Technically the programs entail careful original planning and organization to provide for (a) establishment of pilot projects in which methods are tested for their local suitability, (b) an attack phase in which DDT or other comparable insecticide is applied to houses universally throughout a country and the resultant insecticidal cover maintained for a number of years, $(c)$ a consolidation phase during which the insecticide is discontinued and remaining cases of infection are sought by screening the whole population, and finally $(d)$ establishment of eradication, followed by watchful maintenance till the risk of reinvasion disappears through worldwide eradication.

The attack phase is normally regarded as the process of eradication itself, though it is in reality no more than elimination of the branches and the ending of the widespread proliferation of malaria throughout the country. These accomplishments make it possible during the consolidation phase to institute a search for the mother foci on which the proliferation was dependent. Even very recently there were no acknowledged criteria as to what should happen to malaria during this process. Thus, appraisal of schemes in their early operation was not possible except in the most indecisive terms. Only after some years could the end product of virtual disappearance or obvious continuation be seen. Nor were there any objective criteria for recognizing the stage when it was reasonable to discontinue spraying and substitute alternative techniques of casefinding. Inevitably attack must in some instances have been discontinued too early. The techniques of searching for cases also had to be elaborated. Moreover, the ultimate task of proving a zero incidence in a vast population presents great difficulties.

Considering these difficulties, the general scale of achievement is remarkable. Any criticism and comment must be understood to apply to relatively small facets of what is on the whole a brilliantly executed operation. Most schemes have been undertaken with determination. The technical effort of the Organization in support of eradication has been imaginative and great 
in scale, and most programs are progressing with perhaps even more success than could have been rationally forecast some years ago.

\section{Technical Difficulties with Malaria}

Nevertheless there have been technical difficulties. Objectivity, long lacking in the appraisal of the pilot project and of the attack phase has now at last been provided. The rate of disappearance of infection in a series of cases studied some 25 years ago provided a clue as to objectivity. When the cases were shown on ratio paper, the rate of disappearance was seen to have an exponential form with a half-life of about 140 days. Study of several known successful schemes showed that, once malaria transmission was decisively ended, cases in fact diminished in this same exponential manner. This was the way cases decreased during the eradication of $A$. gambiae from Brazil (2), in the successful Taiwan eradication project (12) (fig. 1), and in all other projects examined. Criteria were established in 1964 (11) of the expected rate of decrease and the minimum tolerable rate of decrease. These criteria give reality to appraisal even in the early days of attack and for pilot projects. They can be supported by other equally objective criteria for widespread application. Thus, not only can progress be evaluated, but the probable dates of achievement of adequately low levels can be forecast. Criteria for safe withdrawal of attack have been improved by analysis of the consequent load thrown on epidemiologic staff in providing sufficient study of all residual cases found. There is still room, however, for application of statistical techniques to evaluation of minor divergences from the expected in the numbers found.

The next group of technical difficulties was presented by the mosquito. Resistance has been mentioned, and its nature and method of selection have been understood for some years. The data collected have often made it possible to avoid the consequences of resistance to one pesticide by use of alternatives. In the meantime, research, which is now on a large scale, is producing alternative materials at least as fast as the mosquito is likely to adapt itself to existing ones. For management by means other than pesticides, current research on genetic manipulation holds out the most interesting possibilities.

There are other problems which, although locally important, are relatively small in relation to the general picture. One of these problems concerns mosquitoes which either habitually avoid houses or do so when treated with insecticides. Solution of this difficulty is now engaging some of the best minds working on malaria eradication. Eradication, however, need not confine itself to the traditional techniques of standard insecticides. It is able to use any form of attack, including mass chemotherapy with medicated salt. Therefore there

Figure 1. Rate of decease of Plasmodium falciparum parasite rates following total interruption of transmission in Brazil and Taiwan

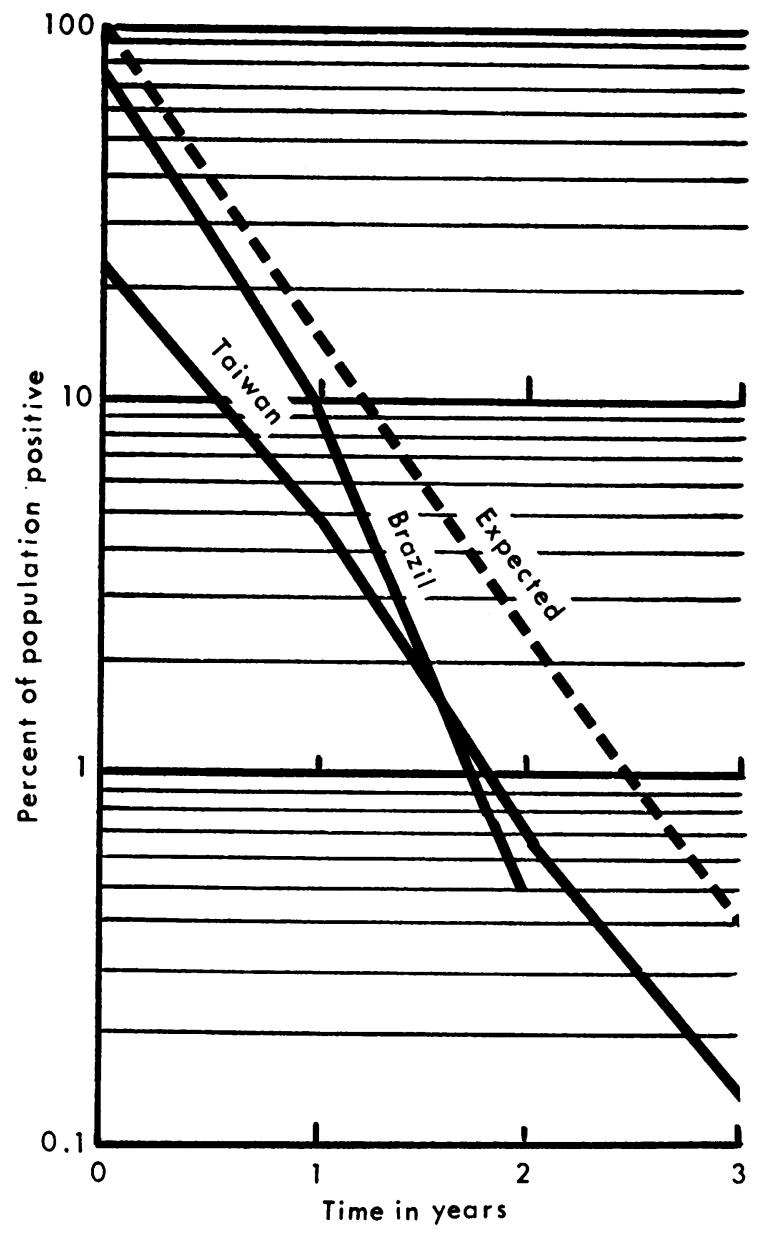

Source: References 2 and 12. 


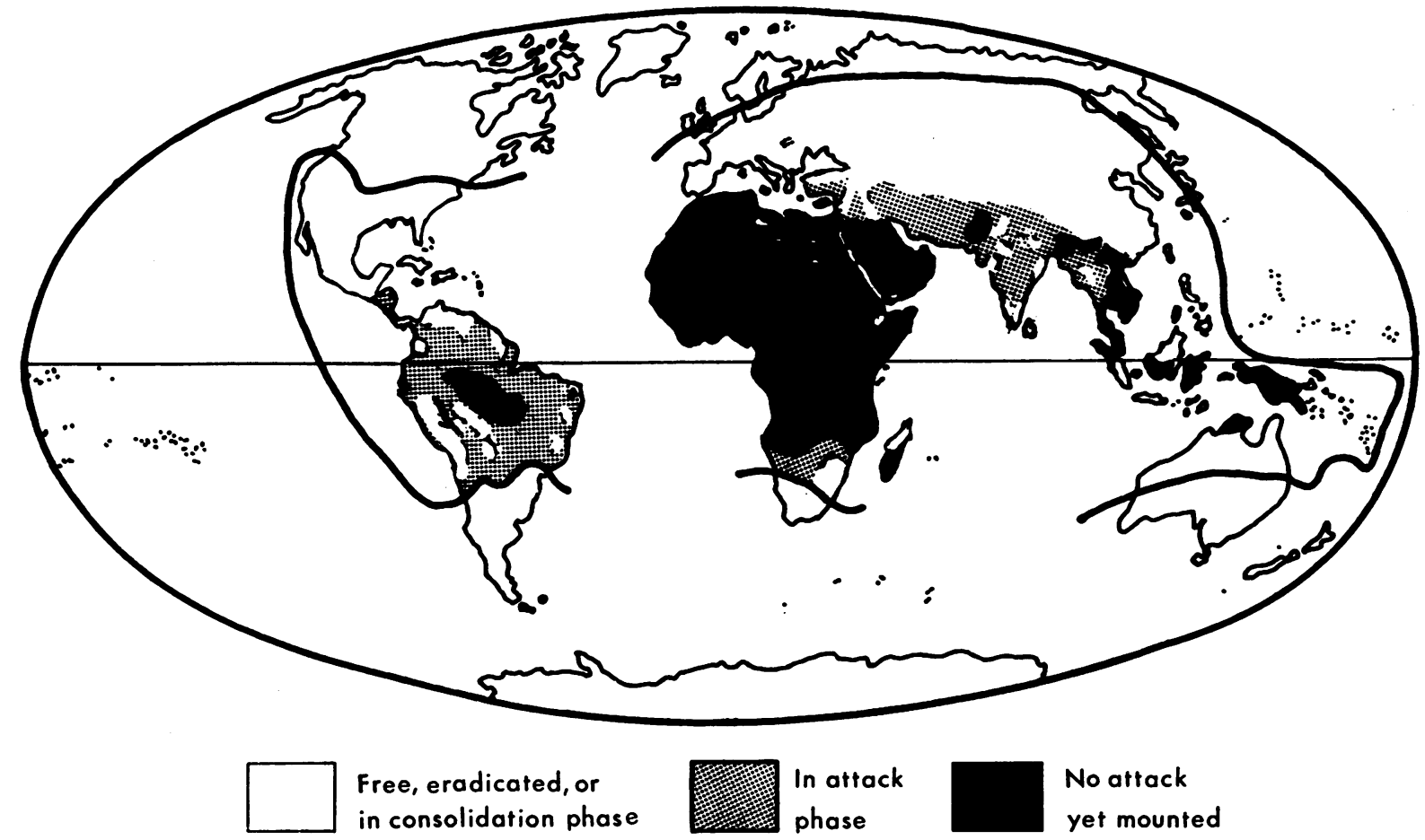

\section{Previous limits of prevalence}

Nore: "No attack" areas in Brazil are shown to the correct scale but are not necessarily correctly located, owing to lack of precise information.

is no reason to think that such difficulties amount to anything more than severe local setbacks. They can be overcome by use of resources at our disposal. Since they occur in areas which, in comparison with the total malarious areas of the world, are small, a larger utilization of skills and efforts is entirely feasible.

Against these problems must be set the exceptional degrees of success achieved either by modification of method or by locally favorable biological events. Extremely rapid reduction of the parasite reservoir has been achieved in some experimental areas by simultaneous administration of drugs to the population and application of insecticides, for example, in Kigezi in Uganda. Another exceptional degree of success occurred in a pilot project in Liberia, where the anopheline vector was totally eradicated. In some areas of South and Central Africa, what had been thought to be one widespread species of $A$. gambiae was in reality at least two subspecies. Only one was significant in transmission of malaria, and it has proved readily amenable to complete control.

Technically, therefore, the prospects for eradication of malaria are bright. Administrational difficulties still arise, but mainly in the consolidation stage, when the process of surveillance is applied. This stage constitutes the search for and destruction of the roots of the infection after the branches have been destroyed. It requires an extremely detailed search throughout the entire population for any remaining cases or foci of cases and then the application of fully effective treatment, using whatever other remedies epidemiologic study has shown to be necessary. The process calls for repeated examination of blood films from a considerable proportion of the population and maintenance of a laboratory study disseminated over a wide area and of operational personnel or groups able to make an imaginative study and to react rapidly to their findings. The dispersion of these skills and the maintenance 
of an even cover to keep an entire population under watch taxes any administrative structure and presents a major problem of eradication.

\section{Progress in Malaria Eradication}

Despite these difficulties, general progress has been good. Of 1,472 million persons previously exposed to the risk of malaria, 572 million are now free from it because of campaigns that have reached the consolidation phase. An additional 461 million are covered by campaigns still in the attack phase. Such progress is at first glance, satisfying, but complacency cannot survive a detailed examination of findings. Figure 2, prepared from official WHO figures for 1963 (13), shows progress on an isometric- scale map. Boundary lines indicate the past limits of malarial prevalence. White areas within these boundaries are free from malaria by reason of its natural disappearance, its eradication, or entry of the eradication program into the consolidation phase. Shaded areas are those protected by the attack phase. The black areas are those in which attack has not yet started. A marked geographic bias is clear. A great recession from the original limits, as well as generally throughout temperate and subtropical regions, is encouraging. A vast scheme in India quickly approaches success, and energetic schemes exist in all American countries. Africa and parts of the Middle East are, however, almost untouched.

The geographic bias is made even more not-

Figure 3. Progress in malaria eradication among populations at risk, by World Health Organization regions, in areas of no eradication attack, areas of attack, and areas of consolidation or maintenance, 1962

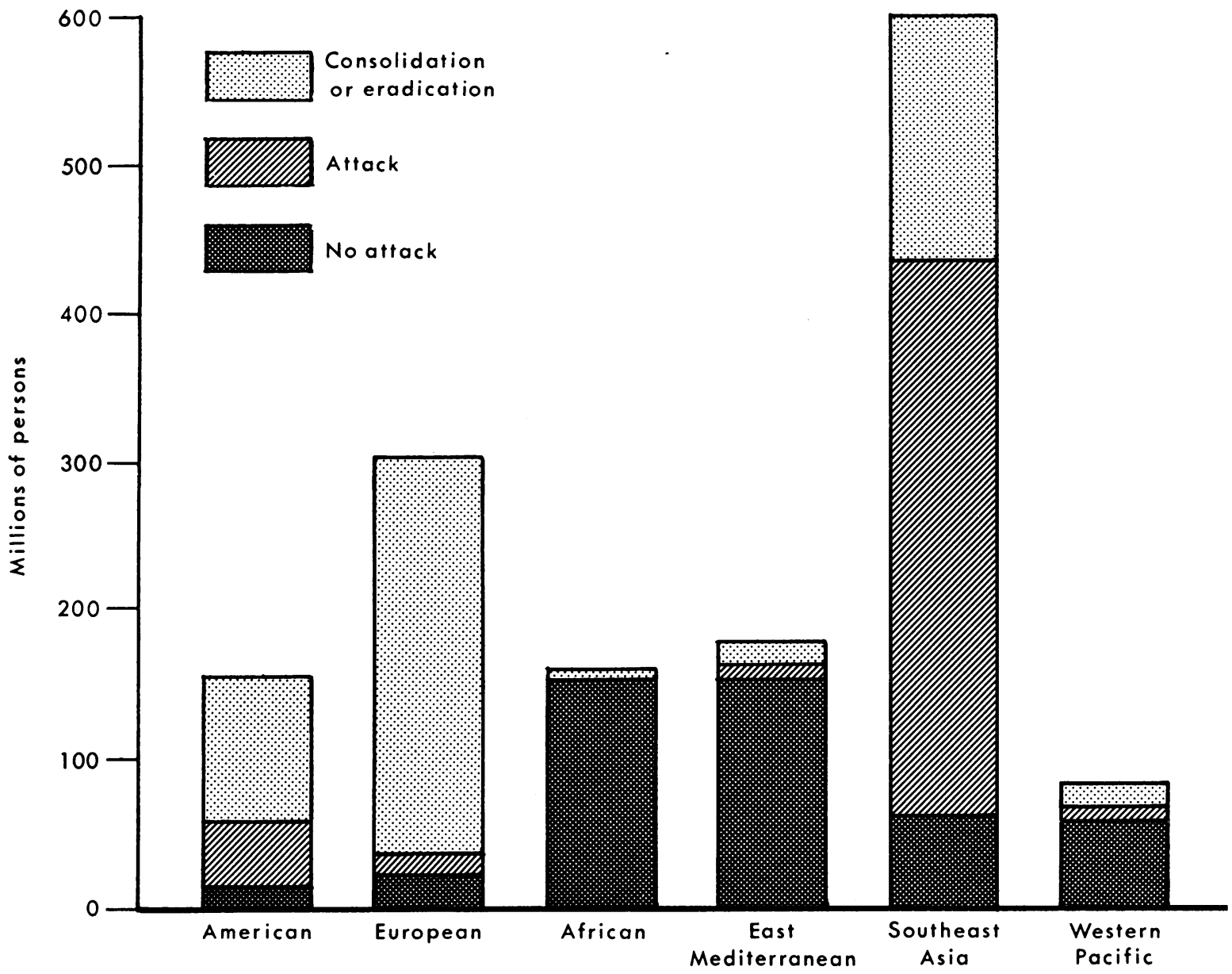




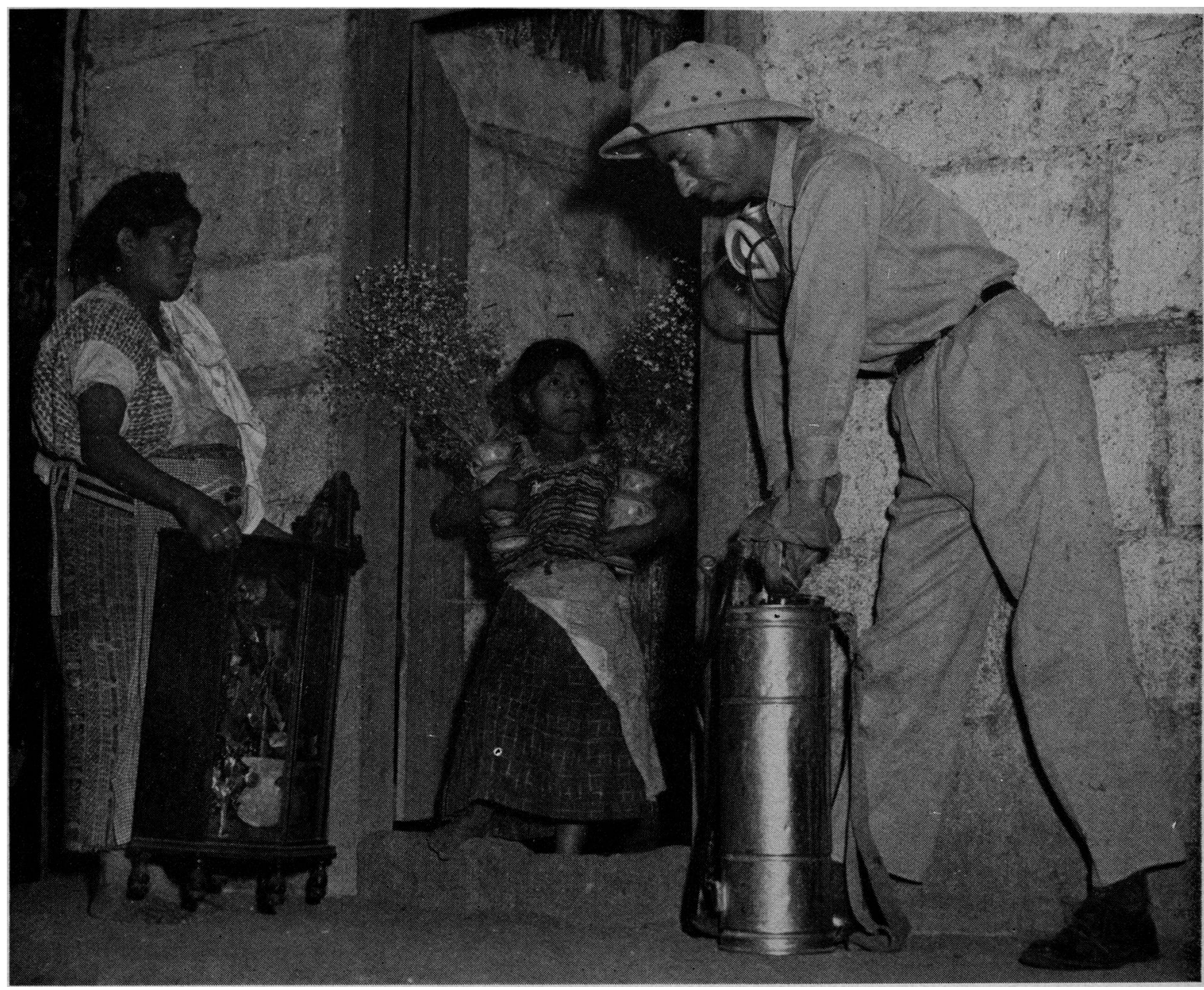

Guatemala: Mother and daughter remove valued pieces from their home before it is sprayed by a member of the malaria eradication team. The rural, thatched-roof adobe houses are excellent places for the harboring of anopheline mosquitoes.

able by examination in individual WHO regions of the populations at risk and the degree of their protection (fig. 3). The African and Eastern Mediterranean regions stand out as ones in which eradication has still effectively to start. There are many reasons for this. Certainly Africa presents greater technical problems than any other land, its administrative systems are poorer, and its sources of skill are shallower. But are these now adequate reasons for failure to start eradication?

\section{Obstacles to Malaria Eradication}

The four types of obstacles to be overcome are: inertia to change the agelong position; resistance of mosquitoes to insecticides or of parasites to drugs; incapacity to stage an effective program; and inaccessibility of people or of mosquitoes. Incapacity is the most readily demonstrated and most frequently avowed reason for postponement. Resistance and inaccessibility present technical and operational difficulties which can be overcome. Inertia has obstructed progress against malaria in Africa for many years. It can take advantage of other difficulties to demand further research and to explain delay. Inertia has to be overcome in ourselves and in the individual countries before Africa can start to catch up with the other WHO regions.

Undoubtedly incapacity exists side by side with inertia, and often as a partial explanation. Incapacity, however, is never more than tempo- 
rary. Highly technical skills are needed but not on a great scale, and with the advantages of international and national aid they can be applied to any country. The attack phase demands the deployment of considerable numbers of trained operators. However, because their activities can be highly standardized, a drill for training operators can be developed so that their numbers can be multiplied rapidly in a specific community. The main incapacities really arise in the later phases of extermination of the root. They lie in the difficulty of maintaining a universally distributed staff in close contact with every person in the community, a staff able to take the initiative and backed by a laboratory organization. In a country with virtually no trained technicians and no disseminated rural health centers or even an organized system of local government, this incapacity may be considerable. Overcoming it may demand introduction of training mechanisms for technicians, together with the development of a disseminated health structure, so that sufficiently close contact can be maintained with the population.

Examination of the time scale for creation of a cadre of persons capable of carrying out the function of malaria eradication suggests that, if the need were to train them for this function alone, training could be easily accomplished within the usual period of an eradication campaign. However, since the cadre's duties of guarding against reinvasion must continue after eradication, these persons must still, when malaria has disappeared, have functions that, by keeping them in close touch with the population, will enable them to discover any imported cases of infection. They must, in fact, be able to provide at least the elements of a health service and to provide this throughout the entire country. Such responsibilities require somewhat more elaborate training than would be required for the basic functions of malaria eradication alone. As a result, the training period might be slightly longer than in an eradication program alone. The difference need not be great if the same urgency is put into both aspects of the program. In some instances, however, it might be necessary to antedate the general training by 3,4 , or, for some circumstances, even 5 years before the formal start of malaria eradication, so that the two programs could properly coincide at a later stage.

To provide training in such instances, the Expert Committee on Malaria (11) has supported the principle of pre-eradication programs for areas where health services are elementary. These programs consist essentially of a preliminary phase before malaria eradication begins in which the first steps toward a rural health service are taken and, particularly, training mechanisms for the staff are brought into being. Inevitably this plan recognizes the need to delay malaria eradication in some areas, particularly in Africa. In my conception, however, the delay is small-3, 4 , or 5 years-and certainly does not amount to substantial deferment, such as transferring eradication from one generation of people to the next. The risk exists, nevertheless, that the forces of inertia might extend what should only be a brief delay into a major postponement.

\section{Completion of Malaria Eradication}

Any country or region which remains highly malarious will by the same token remain backward, progressing slowly in a world in which others progress rapidly, and the gaps will inevitably widen. The malarious countries will be sufficiently ready to make a great effort to eradicate malaria providing that inertia is not encouraged from without. Inertia will have to be replaced by a sense of urgency, assurances of technical feasibility, and sufficient technical and material aid to help the countries mount schemes obviously beyond their immediate means. These assurances can be given. The aid mechanisms would be on no larger scale than are now employed for comparable purposes. The grievous need is for the encouragement to a sense of urgency. Some health workers believe that the fire to eradicate is beginning to die down, now that the already developed or partially developed countries are well on their way to eradication.

The impetus to malaria eradication can be maintained, but it needs to be transferred from the countries which are moderately or well developed to those which have suffered all the disadvantages and in which eradication has been 
slow. These countries need the encouragement and support of training mechanisms so that they can produce an adequate staff not only to carry out malaria eradication but to maintain it once it is achieved. Coordination between the countries themselves is needed. It can be achieved through organizations such as the East African Common Services Organisation or, in the French-speaking territories of West Africa, Organization de Coordination et de Cooperation pour la Lutte contre les Grandes Endémies. With the aid of these coordinating mechanisms, regional programs are required which will cover geographic areas of considerable size. These areas must have conditions which are reasonably homogeneous and boundaries which can be effectively protected against reinvasion of the infection.

Research is needed, although always in parallel to operational schemes. Never, as happened too often in the past, should it consist of preliminary studies which indefinitely delay the start of activity and have no real prospect of solving operational problems. No more preliminary experiments are required. Experimental processes should be incorporated within the mainstream of operational activity. They should be geared to spotting and solving operational problems as they develop and to feeding research findings back for incorporation into standard procedure. Finally, these procedures should be backstopped by central regional, or even central continental, laboratories capable of taking on the solution of the larger problems.

In the last 18 years we have seen the world's malarious areas shrunk by more than half in terms of population affected, despite what once seemed a hopeless outlook. Completion of this process-the eradication of malaria, root and branch-not only from the relatively favored countries but also from the most underdeveloped now seems no more difficult than did even moder- ately effective control 18 years ago. The principle of eradication in contrast with control derives largely from Soper. $\mathrm{He}$ has seen the goal of eradication well advanced, and there is no valid reason why he should not see it reached.

\section{REFERENCES}

(1) Soper, F. L., and Wilson, D. B.: Species eradication: practical goal of species reduction in control of mosquito-borne diseases. J Nat Malar Soc 1 : 5-24 (1942).

(2) Soper, F. L., and Wilson, D. B.: Anopheles gambiae in Brazil, 1930-1940. The Rockefeller Foundation, New York City, 1943.

(3) Shousha, A. T.: Species eradication; eradication of Anopheles gambiae from Upper Egypt, 194245. WHO Bull 1 : 309-348 (1948).

(4) Soper, F. L. : Problems to be solved if the eradication of tuberculosis is to be realized. Amer J Public Health 52 : 734-748, May 1962.

(5) Karunaratne, W. A.: The influence of malaria control on vital statistics in Ceylon. $J$ Trop Med Hyg 62: 79-85, April 1959.

(6) Davidson, G.: Studies on insecticide resistance in anopheline mosquitoes. WHO Bull 18 : 579-621 (1958).

(y) World Health Organization Expert Committee on Malaria : Sixth report. Technical Report Series No. 123. Geneva, 1957.

(8) World Health Organization Expert Committee on Malaria: Seventh report. Technical Report Series No. 162. Geneva, 1959.

(9) World Health Organization Expert Committee on Malaria: Eighth report. Technical Report Series No. 205. Geneva, 1961.

(10) World Health Organization Expert Committee on Malaria: Ninth report. Technical Report Series No. 243. Geneva, 1962.

(11) World Health Organization Expert Committee on Malaria: Tenth report. Technical Report Series No. 272. Geneva, 1964.

(12) Taiwan Provincial Malaria Research Institute and World Health Organization malaria team in Taiwan: Malaria control and eradication in Taiwan, progress report, May 1952 to June 1957. WHO Bull 19 : 595-620 (1958).

(13) World Health Organization: Official Records No. 127. Geneva, 1963. 